\title{
Mechanical properties of compression molded banana pseudo-stem filled unplasticized polyvinyl chloride (UPVC) Composites
}

\begin{abstract}
Mechanical strengths of a banana pseudo-stem (BPS) fiber and unplasticized polyvinyl chloride (UPVC) composite were evaluated to assess the possibility of using it as a new material in engineering applications. Samples were fabricated by the compression molding process with reference to the effect of filler loading. The samples were submitted to mechanical tests to measure tensile, flexural, and impact properties of the composites. The nature of adhesion between the matrix and the reinforcement and information relating the structure of mechanical properties can be obtained by scanning electron microscopy (SEM) assessment of the composite fracture surface. The mechanical properties show that the composites did not have good adhesion between filler and matrix; on the other hand, the filler insertion improved the flexural modulus and the material rigidity.
\end{abstract}

Keyword: Banana filler, Flexural property, Impact property, Tensile property 